\title{
Assessment of efficiency of innovative technologies in tourism
}

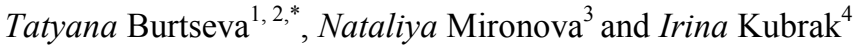 \\ ${ }^{1}$ Vyatka State University, 36, Moskovskaya str., 610000, Kirov, Russia \\ ${ }^{2}$ Moscow University named after S.Yu. Witte, 12, bld.1, 2nd Kozhukhovsky passage, 115432, \\ Moscow, Russia \\ ${ }^{3}$ Perm State Institute of Culture, Faculty of Cultural Studies and Socio-Cultural Technologies, 18, \\ Gazeta Zvezda St., 614000, Perm, Russia \\ ${ }^{4}$ Moscow Region State University, 10A, Radio str., 105005, Moscow, Russia
}

\begin{abstract}
The article describes the main innovative technologies that are successfully applied today in the field of tourism. Approaches to assessing the effectiveness of innovative technologies is especially important in the system of managing the competitiveness of companies in the tourism sector. There are many methods for assessing effectiveness, both quantitative and qualitative methods. This article proposes the method for qualitative assessment of the effect of the use of innovative technologies, such as "Internet maps", which are used as a reference tool and a guide for general acquaintance with the travel area, and "Organizational resources", including electronic reservation tools and electronic payment methods., to the level of corporate image of a tourist company. The assessment is based on the use of the analysis of variance of the research data.
\end{abstract}

\section{Introduction}

Innovation is an implemented improvement that provides a qualitative increase in the efficiency of processes or products demanded in the market. Technology is the totality of all approaches, methods, developed tools that the company uses to achieve a specific goal and solve practical problems. Therefore, in our opinion, innovative technologies are ways of working, mode, sequence of actions how to use innovations in order to achieve goals and solve urgent problems of the company.

The more developed the market, the shorter the life cycle of a product or service. Therefore, it is very difficult to effectively promote any activity without the introduction of new technologies and scientific achievements, improvement of existing models considering changes in the market and customer needs, without the ability to be flexible. Such changes determine the innovative development way of the industry[1-3].

«Innovations in tourism are novelties and fresh ideas in the field of travel and leisure. The result of the introduction of such innovations is an increase in tourist flow and profit growth. Each industry has its own characteristics, the success of its development depends

\footnotetext{
*Corresponding author: burtseva_10@mail.ru
} 
on them. The income from tourism is the main source for residents of some countries or it makes up a significant part of the state budget» [4].

The main innovative activities in tourism are:

1. Implementing advertising campaigns (through the media).

2. Attracting consumers through discounts and promotions and sending this message to tourists.

3. Creating a positive reputation with potential customers, improving the service, forming a feedback system about your company [5].

4. Using information technology, including the development and creation of convenient means of searching and delivering information, sites, mobile applications, etc.

5. Implementing and promoting new types of services in the region: new tours, routes, cooperation with airlines, hotels, restaurants, visiting museums via the Internet and more [6].

6. Building new tourist facilities.

7. Studying the work of competitors, including successfully developing ones [4].

8. Using "Electronic Offices", which allow to book various travel services (hotels, tickets for recreational activities, excursions and others).

9. Using virtual concierge, this innovation is implemented in 12 hotels of the UK.

10. Cinema tourism - organization of excursion tours to places of filming famous movies.

11. Mobile hotels that allow to live in one place while traveling around the country.

12. Ecotourism. This relatively new type of vacation involves tourists staying in a private house or in a family countryside, surrounded by nature.

13. Virtual museums - visiting the expositions of famous museums via the Internet.

14. Internet cards, which serve as a reference tool and a guide for general overview, exploring the travel area, obtaining the necessary information about the location of sightseeing places.

15. Organizational resources include electronic reservations and electronic means of payment. The interaction of the tour operator with booking information systems creates the following opportunities: expanding the market for tourism products; forming a list of consumers; operational management of their activities; analysis of demand for tourism products; marketing planning and more. For travel agencies, reservation systems are a necessary tool for providing customers with high-class services [2].

\section{Methods and Materials}

As for managing the economic efficiency of using innovative technologies in the tourism industry, first of all, a methodological and methodological apparatus is of great importance to evaluate managerial decisions. It is not always advisable to estimate based on numerical values a qualitative assessment is also important. In the system of marketing research, a qualitative assessment of effectiveness is used quite widely and has its own advantages and disadvantages.

One of the interesting approaches to a qualitative assessment of the effectiveness of managerial decision-making is analysis and evaluation based on using the variance analysis of research data.

Let us consider the technology for assessment of efficiency of innovative technologies in tourism.

Take the example of assessing the efficiency of using the "Internet card" as a reference tool and a guide for general information with the travel area (X1) and "Organizational resources", including electronic reservation tools and electronic payment methods $(Z)$, and its influence the image of a tourist company (Y). 
To assess the qualitative effect of the influence of an Internet card (X1) and organizational resources on the corporate image level, we carried out the research on the tourist services market. The data are presented in Table 1. The assessment is based on the analysis of variance, which is used to study the differences in the average values of dependent variables. These variables include the level of corporate image, caused by the influence of controlled independent variables, the presence or absence of Internet cards in the company and the use of organizational resource.

Table 1. Tourism Market Research Results.

\begin{tabular}{|c|c|c|c|}
\hline $\begin{array}{c}\text { Objects of study } \\
/ \\
\text { Observations }\end{array}$ & $\begin{array}{c}\text { Corporate image level, } \\
\text { points }\end{array}$ & $\begin{array}{c}\text { Internet cards } \\
1 \text { - no } \\
2 \text { - yes }\end{array}$ & $\begin{array}{c}\text { The range of organizational } \\
\text { resources } \\
1 \text { - wide } \\
2 \text { - medium } \\
3 \text { - limited }\end{array}$ \\
\hline 1. Company A & 9 & 2 & 3 \\
\hline 2. Company B & 8 & 2 & 3 \\
\hline 3. Company $\mathrm{C}$ & 9 & 2 & 3 \\
\hline 4. Company D & 9 & 2 & 2 \\
\hline 5. Company E & 6 & 2 & 2 \\
\hline 6. Company $\mathrm{F}$ & 3 & 2 & 2 \\
\hline 7. Company G & 4 & 2 & 1 \\
\hline 8. Company $\mathrm{H}$ & 4 & 2 & 1 \\
\hline 9. Company I & 5 & 2 & 1 \\
\hline 10. Company $\mathrm{G}$ & 5 & 1 & 1 \\
\hline 11. Company K & 2 & 1 & 2 \\
\hline 12. Company L & 3 & 1 & 2 \\
\hline 13. Company M & 2 & 1 & 3 \\
\hline 14. Company N & 3 & 1 & 2 \\
\hline 15. Company O & 2 & 1 & 1 \\
\hline 16. Company P & 2 & 1 & 3 \\
\hline 17. Company Q & 4 & 1 & 3 \\
\hline 18. Company R & 4 & 1 & 1 \\
\hline
\end{tabular}

\section{Results}

To study the effect of variables on sales, we took 18 travel agencies. For each combination of conditions, we took six companies with and without a franchise, 3 companies with a wide, medium and limitied range of organizational resources. The corporate image level was determined on a 10-point scale; quantitative indicators were operationalized (translated into points) for the analysis procedure.

The main stages of determining effectiveness using the analysis of variance are:

1. At the first stage, we assess the influence of the breadth of application of organizational resources on the level of the corporate image of the company using the software product for statistical processing of the Minitab research data. Based on the oneway analysis of variance, we determine the differences in the average values of the dependent variable for several categories of the independent variable to see if the level of corporate image (Y) depends on the breadth of application of organizational resources (X1), (high, medium and low) [3].

2. Then we formulate the null hypothesis, which states that group averages for tourism companies do not depend on the breadth of application of organizational resources (X1), i.e. are equal: But: $\mu 1=\mu 2=\mu 3$ 
3. To determine the strength of the influence of the variable $\mathrm{X}$ on $\mathrm{Y}$, we determine the indicator $\mathrm{\eta} 2: \mathrm{\eta} 2=\mathrm{SSx} / \mathrm{SSy}=(\mathrm{SSy}-\mathrm{SSerror}) / \mathrm{SSy} ; \mathrm{\eta} 2=0$, when all group means are equal, i.e. the variable $\mathrm{X}$ does not influence $\mathrm{Y} ; \mathrm{y} 2=1$, when within each group of the variable $\mathrm{X}$ there is no variability, but there is some variability between the groups [3].

The we determine the influence of the breadth of the range of products $(\mathrm{X})$ on sales $(\mathrm{Y})$. The degree of influence is $\mathrm{y} 2=\mathrm{SSx} / \mathrm{SSy}=9,33 / 108,00=0,0864$ i.e. $8,64 \%$ of the variation in the level of corporate image (Y) is due to the influence of the breadth of application of organizational resources, which indicates a very low level of influence.

4. To check the statistical significance of the influence, we use the following criteria: Fisher F-test, Tukey test and graphic tests.

Table 2. The results of a one-way dispersed analysis of the impact of the breadth of application of organizational resources on the level of corporate image.

\begin{tabular}{|c|c|c|c|c|c|}
\hline Variance source & \begin{tabular}{|c|} 
Sum of squares, \\
SS
\end{tabular} & $\begin{array}{l}\text { Degrees of } \\
\text { freedom, } d f\end{array}$ & $\begin{array}{c}\text { Average } \\
\text { square, } M S\end{array}$ & $\mathbf{F}$ & $\begin{array}{c}\text { Probability } \\
\text { P }\end{array}$ \\
\hline $\begin{array}{l}\text { Between groups } \\
\text { (the breadth of } \\
\text { application of } \\
\text { organizational } \\
\text { resources) }\end{array}$ & 9.33 & 2 & 4.67 & 0.71 & 0.508 \\
\hline $\begin{array}{l}\text { Inside groups } \\
\text { (variance of error) }\end{array}$ & 98.67 & 15 & 6.58 & - & - \\
\hline Total & 108.00 & 17 & - & - & - \\
\hline & \multirow{2}{*}{\multicolumn{2}{|c|}{ Number of observations }} & \multicolumn{3}{|l|}{ Average } \\
\hline $\begin{array}{l}\text { The breadth of } \\
\text { application of } \\
\text { organizational } \\
\text { resources }\end{array}$ & & & Average & Stan & ard deviation \\
\hline High level (1) & \multicolumn{2}{|c|}{6} & 4.000 & \multicolumn{2}{|r|}{1.095} \\
\hline Middle level (2) & \multicolumn{2}{|c|}{6} & 4.333 & \multicolumn{2}{|r|}{2.658} \\
\hline Low level (3) & \multicolumn{2}{|c|}{6} & 5.667 & \multicolumn{2}{|r|}{3.386} \\
\hline Total & \multicolumn{2}{|c|}{18} & - & \multicolumn{2}{|r|}{-} \\
\hline
\end{tabular}

4.1 To test the null hypothesis about the equality of average values at different levels of the breadth of application of organizational resources, the values of F-calculated, which is in our case 0,71 , compare with the critical value of the $\mathrm{F}$ in the table. For 2 and 17 degrees of freedom, the tabular value is $F=3,59$, which is less than the calculated value. Therefore, the null hypothesis about the equality of the corporate image level is not rejected and the effect of the influence of organizational resources on the corporate image level is not statistically significant.

4.2 Tukey test for significance.

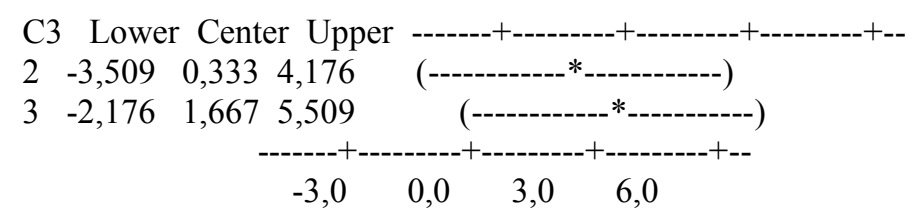

Fig. 1. Turkey test.

According to the Tukey test (Figure 1), intervals (-3.509 to 4.176) were obtained. This means that the difference between the sales volume with an average breadth of the assortment and a high breadth can be in the range from -3.509 to 4.176. Since zero is in this range, the difference in the average values for these two values cannot be considered 
statistically significant. Therefore, we use graphic tests to check the significance of the effect [3].

4.3 Graphic Tests.

4.3.1 We build an individual graph of values to determine the grouping of companies depending on the breadth of the offered assortment (X) (Figure 2).

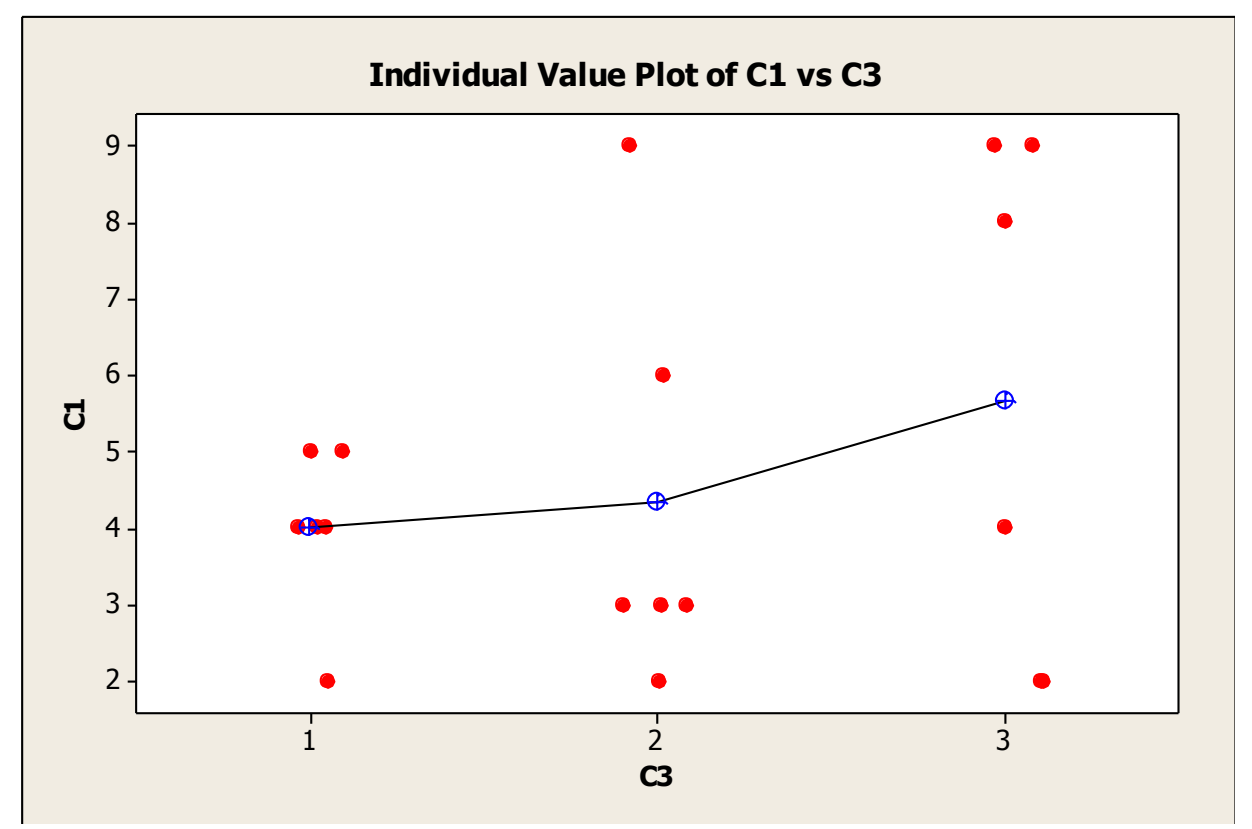

Fig. 2. Individual chart of corporate image level values.

The graph shows the distribution of tourism companies depending on the breadth of application of organizational resources and what level of corporate image each level provides. There is a clear distribution of companies into groups. It can be seen that there is no difference in the level of corporate image with a high and medium level of breadth for the use of organizational resources. The level of corporate image, on the contrary, is high with a low latitude for the use of organizational resources.

4.3.2 Let us build a graph of rectangular diagrams (Figure 3). 
Boxplot of C1 by C3

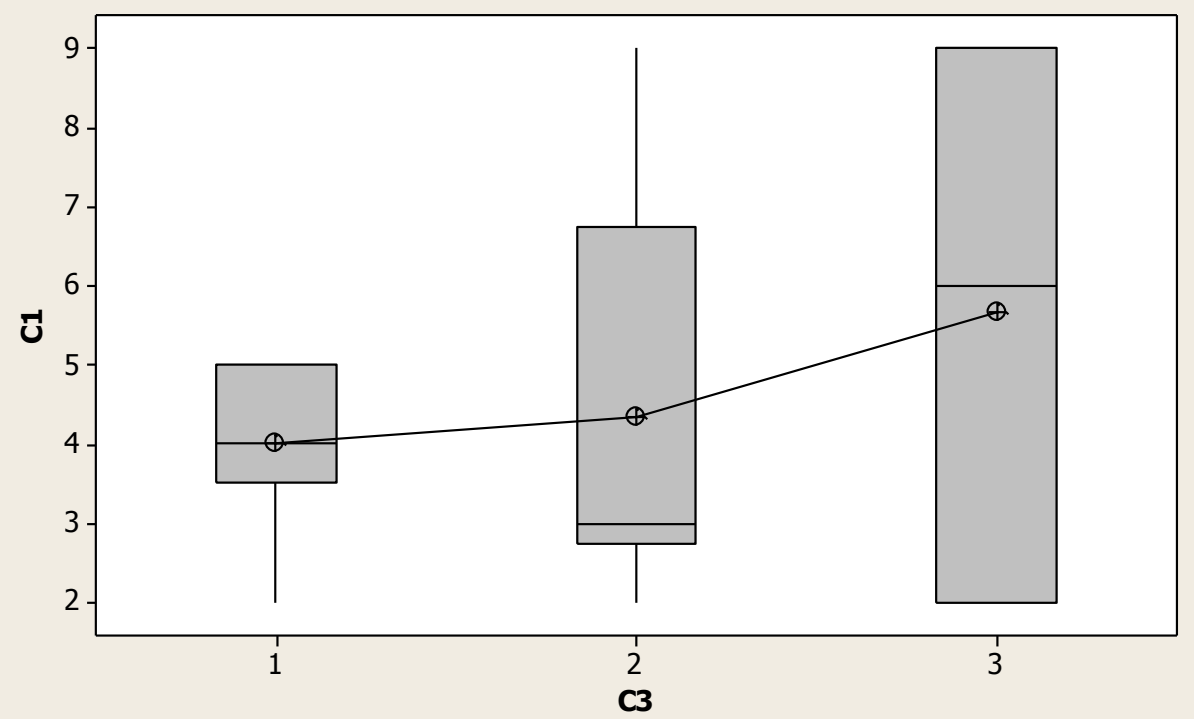

Fig. 3. A rectangular diagram of the dependence of the corporate image level on the breadth of application of organizational resources.

The rectangular diagram shows the level of corporate image at various levels of the dependent variable. The diagram also indicates that the higher the breadth of application of organizational resources is, the higher the level of corporate image.

4.3.3. We build graphs of residues (Figure 4). For the analysis, we use the statistical indicator "residuals". It is the difference between the observed correlations (data in the original correlation matrix) and the calculated ones, which are estimated based on the factor load matrix. Charts of balances can be used to test the null hypothesis about the equality of average sales volumes (But: $\mu 1=\mu 2=\mu 3$ ). 


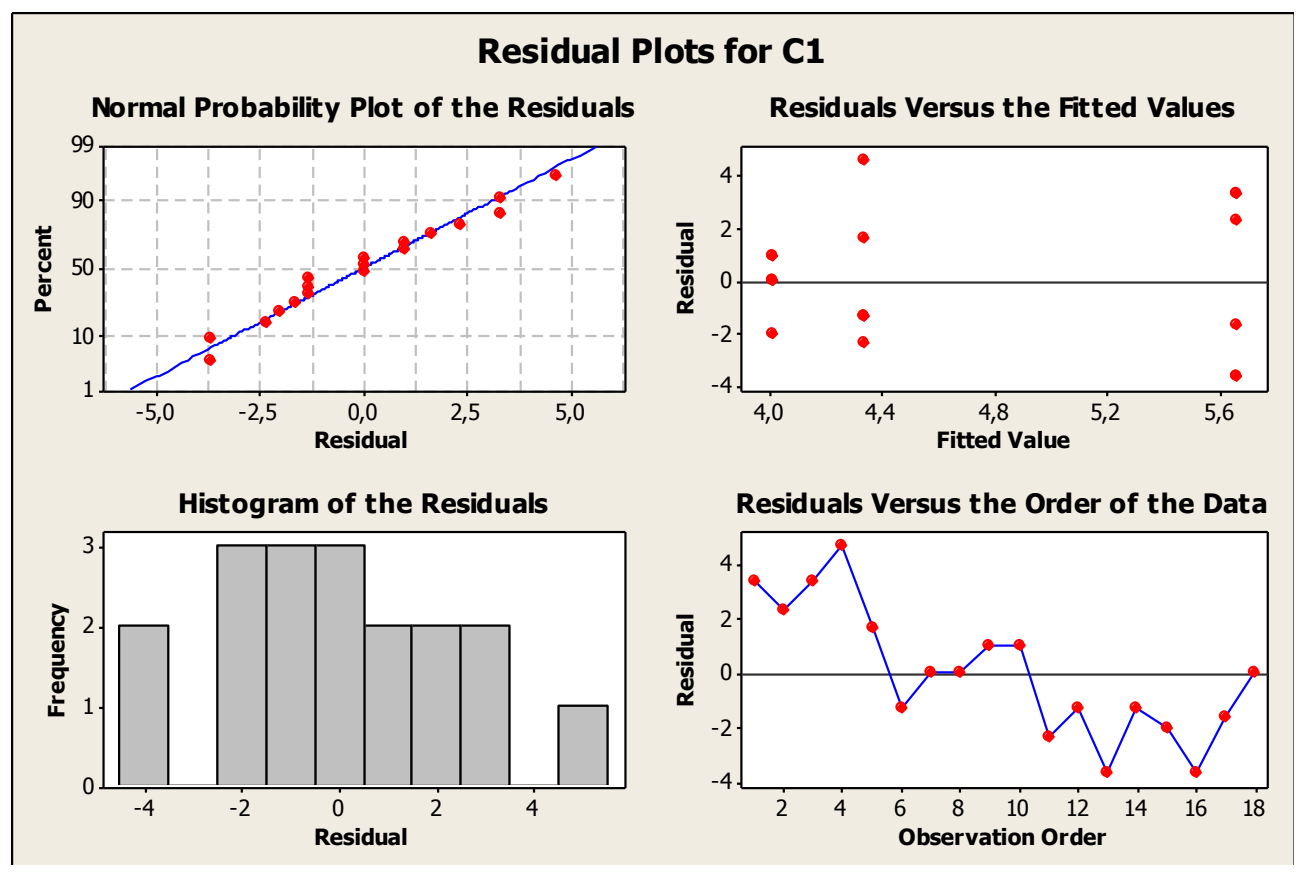

Fig. 4. Graphs of residuals to test the null hypothesis.

If the graph is close to a straight line, as in our case, then the residuals are distributed normally and the null hypothesis of equality of mean values is rejected. The Fitted Value graph allows to determine the dependence of residuals on level X. Residues should be located along the Residual axis and not be grouped around the zero axis. The histogram of residues allows to detect the presence of several (peaks) outliers, as well as to reveal nonGaussianity. The histogram should be approximately symmetrical and have the shape of a bell. A graph of residuals and fitted values (Observation Order) allows to identify inconsistent variance, the absence of higher order members and the presence of outliers. Residues should be randomly located around zero. As can be seen from the graphs of the balances, the null hypothesis is also not rejected, and the breadth of application of organizational resources does not significantly affect the level of corporate image of the travel agency.

5. To determine how two dependent variables - the breadth of organizational resources (Xi) and the presence or absence of an Internet card ( $\mathrm{Zi}$ in influence the corporate image level (Yi), we use the two-way analysis of variance. The results of the analysis are presented in Table 3.

6. Determination of the full effect (full correlation), i.e. degrees of combined influence: $\mathrm{y} 2=(\mathrm{SSx} 1+\mathrm{SSx} 2+\mathrm{SSx} 1 \mathrm{x} 2) / \mathrm{SSx} 1+\mathrm{SSy}=(50,00+9,33+21,333) / 108,00=0,7468$ or $74,68 \%$, which means a rather strong effect of influence.

7. The null hypothesis is checked using F - statistics:

$\mathrm{F}$ is the criterion for checking the significance of the full effect. It is:

$\mathrm{F}=(\mathrm{SSx} 1 \times 2 / \mathrm{dfn}) /(\mathrm{SSerror} / \mathrm{dfd})=\mathrm{MSx} 1 \times 2 /$ MSerror $,=16,133 / 2,278=7,08$

with 5 and 12 degrees of freedom. Fcrit $=3,11$, therefore, the full influence of the breadth of organizational resources $(\mathrm{Xi})$ and the Internet card $(\mathrm{Zi})$ on the corporate image level (Yi) is statistically significant at a significance level of 0,05 . 
Table 3. Two-way analysis of variance.

\begin{tabular}{|c|c|c|c|c|c|}
\hline Variance source & $\begin{array}{c}\text { Sum of } \\
\text { squares. SS }\end{array}$ & $\begin{array}{c}\text { Degres of } \\
\text { freedom. } d f\end{array}$ & $\begin{array}{c}\text { Average } \\
\text { square. } M S\end{array}$ & $\mathrm{~F}$ & $\mathrm{~F}_{\text {crit }}$ \\
\hline $\begin{array}{c}\text { Internet card } \\
\text { availability }\end{array}$ & 50.00 & 1 & 50.00 & 21.95 & 4.75 \\
\hline $\begin{array}{c}\text { Breadth of application } \\
\text { of organizational } \\
\text { resources }\end{array}$ & 9.333 & 2 & 4.667 & 2.05 & 3.89 \\
\hline Combined & 59.333 & 3 & 19.776 & - & - \\
\hline Two-way interaction & 21.333 & 2 & 10.667 & 4.68 & 3.89 \\
\hline Model & 80.666 & 5 & 16.133 & 7.08 & 3.11 \\
\hline $\begin{array}{l}\text { Residual component } \\
\text { (error) }\end{array}$ & 27.333 & 12 & 2.278 & - & - \\
\hline Total & 108.000 & 17 & 6.352 & - & - \\
\hline $\begin{array}{c}\text { Breadth of application } \\
\text { of organizational } \\
\text { resources }\end{array}$ & $\begin{array}{c}\text { Internet card } \\
\text { availability }\end{array}$ & $\begin{array}{l}\text { Number of } \\
\text { observations }\end{array}$ & \multicolumn{2}{|c|}{ Average } & \\
\hline High & - & 6 & \multicolumn{2}{|c|}{4.000} & \\
\hline Middle & - & 6 & \multicolumn{2}{|c|}{4.333} & \\
\hline Low & - & 6 & \multicolumn{2}{|c|}{5.667} & \\
\hline- & Yes & 9 & \multicolumn{2}{|c|}{6.333} & \\
\hline- & No & 9 & \multicolumn{2}{|c|}{3.000} & \\
\hline
\end{tabular}

$\mathrm{F}$ - the criterion for checking the significance of the interaction effect is:

$\mathrm{F}=10,667 / 2,278=4,682 \mathrm{c} 2$ and 12 degrees of freedom. Fcrit $=3,89$, the interaction effect is statistically significant, i.e. the impact of the breadth of organizational resources (Xi) and the impact of having an Internet card ( $\mathrm{Zi}$ ) also depend on each other, and a synergistic effect is present.

Assessment of the significance of the main effects:

F - the criterion for checking the significance of the main effect of the breadth of application of organizational resources is: $\mathrm{F}=2,05$, with 2 and 12 degrees of freedom. Fcrit $=3,89$, the main effect of using organizational resources on the level of corporate image, as we noted earlier, is statistically insignificant.

$\mathrm{F}$ - the criterion for checking the significance of the main effect of having an Internet card is: $F=21,95$ with 1 and 12 degrees of freedom. Fcrit $=4,75$. The main effect of using Internet cards by a travel company is statistically significant.

\section{Discussion}

Assessing the effectiveness of innovative technologies in tourism activities means identifying a possible increase in the company's invested funds by involving them in resolving the difficulties associated with the company's activities in the competitive market. The effect is aimed in the future perspective, the effectiveness determines the feasibility of the company in this area. A negative effect means a loss of the desired quality, features, a decrease in potential. A positive effect is likely due to measures taken to reduce the negative effect. The purpose of management is to obtain the greatest effect with minimal costs, this is especially true for the tourist services market, which is characterized by a high level of competition, both in the domestic and foreign markets. And the search and selection of adequate models, approaches, assessment methods is an important decision in the management system in the tourism market $[7,8]$.

A theoretical analysis of studies in the field of performance evaluation allows us to conclude that there are various methods. There are both quantitative and qualitative 
assessment methods that are as close as possible to use in modern Russian conditions. One such approach is estimation using variance analysis.

Thus, the purpose of analysis of variance is to study the presence or absence of a significant influence of any qualitative or quantitative factor, in our case, using the "Internet map" as a reference tool and a guide for general acquaintance with the travel area and "Organizational resources", including electronic means of reservation and electronic means of payment, for changes in the investigated effective attribute (level of the corporate image of the company).

The analysis of variance can be used to solve problems such as 6

- study of the dependence of company income on the type of target market (then gradation classes are consumer segments);

- the dependence of the cost of production per unit of output on the size of the unit of the enterprise (then gradation classes are characteristics of the size of the unit: large, medium, small);

- the dependence of the level of satisfaction with the services of a travel company on the level of qualifications of the company employees and the proposed tourist routes;

- the dependence of the company's income on such factors as the availability of a franchise and the breadth of the proposed range of tourist services, etc.

The tourism industry is subject to global economic trends. The most important trend that allows companies to develop new tourism markets and business models is the widespread penetration of digitalization elements. Digitalization offers technologies, approaches and tools that add value to a tourism product [1].

Table 4. Summary analysis of the assessment of the qualitative effect.

\begin{tabular}{|l|c|l|}
\hline \multicolumn{1}{|c|}{ Indicators } & \multicolumn{2}{|c|}{ Results } \\
\cline { 2 - 3 } & $\begin{array}{l}\text { The degree of influence of } \\
\text { the variables } \boldsymbol{X}_{\boldsymbol{i}} \text { on } \boldsymbol{Y}, \boldsymbol{y}^{2}\end{array}$ & $\begin{array}{c}\text { Conclusion for the } \\
\text { significance level } \mathbf{\alpha}=\mathbf{0 , 0 5}\end{array}$ \\
\hline $\begin{array}{l}\text { 1. The effect of the breadth of } \\
\text { application of organizational resources } \\
\text { and the availability of Internet cards } \\
\text { on the level of corporate image }\end{array}$ & 0,7468 & $\begin{array}{l}\text { The effect is statistically } \\
\text { significant }\end{array}$ \\
\hline $\begin{array}{l}\text { 2. The effect of the breadth of } \\
\text { application of organizational resources } \\
\text { on increasing the level of corporate } \\
\text { image }\end{array}$ & 0,0864 & $\begin{array}{l}\text { The effect is statistically } \\
\text { significant }\end{array}$ \\
\hline $\begin{array}{l}\text { 3.. The effect of the presence of } \\
\text { Internet cards on increasing the level } \\
\text { of corporate image }\end{array}$ & 0,8427 & $\begin{array}{l}\text { The effect is statistically } \\
\text { significant }\end{array}$ \\
\hline
\end{tabular}

A summary analysis (table 4) showed the degree of influence of the variables and their significance. The greatest influence on increasing the level of corporate image development is provided by Internet cards.

\section{Conclusion}

Thus, our research allows to draw the following conclusions:

1. The widespread use of organizational resources does not significantly affect the level of corporate image of the company. The degree of influence of the breadth of the assortment $\mathrm{y} 2=0,0864$, i.e. $8,64 \%$ of the variation in the level of corporate image (Y) is due to the influence of the breadth of application of organizational resources, which indicates a very low effect 
The effect of the company Internet-cards significantly increases the level of corporate image, and statistically significant. $\mathrm{\eta} 2=0,8427$, i.e. $84,27 \%$ of the variation in sales (Y) is due to the influence of the presence of an "Internet card" as a reference tool and a guide for general information about the travel area.

In our example, the effect of each factor depends on another factor. Therefore, when developing measures to increase the efficiency of using innovative technologies in tourism, we should pay attention to the interconnection and mutual influence of the used innovative technologies.

\section{References}

1. N.A. Mironova, Moscow Economic Journal 5 (2020)

2. N.S. Tsyrinova, Young scientist 27.2(131.2), 45 (2016)

3. Application of innovations in the field of tourism. Via Future https://viafuture.ru/katalog-idej/innovatsii-v-turizme

4. How to Interpret Regression Analysis Results: P-values and Coefficients. The Minitab blog https://blog.minitab.com/blog/adventures-in-statistics-2/how-to-interpretregression-analysis-results-p-values-and-coefficients

5. E. Ganebnykh, O. Fokina, T. Burtseva, I. Yanov, MATEC Web of Conferences 106, 08082 (2017)

6. E. Ganebnykh, T. Burtseva, N. Mironova, O. Feoktistova, E3S Web Conf. 110, 01077 (2019)

7. G. Semenova, E3S Web of Conferences EDP Sciences 110 (2019) https://doi.org/10.1051/e3sconf/201911002149

8. V. Holodkova, A. Mottaeva, T. Pokrovskaya, E3S Web of Conferences 164, 11043 (2020) https://doi.org/10.1051/e3sconf /202016411043 